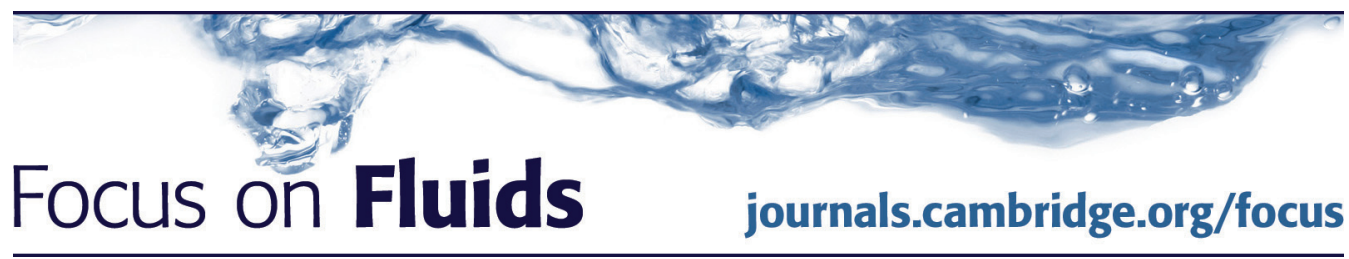

\title{
Short, flat-tipped, viscous fingers: novel interfacial patterns in a Hele-Shaw channel with an elastic boundary
}

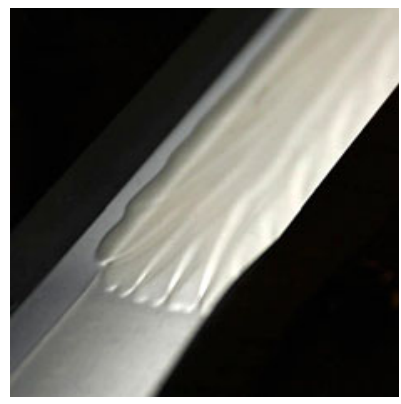

\author{
Scott W. McCue $\dagger$
}

School of Mathematical Sciences, Queensland University of Technology, Brisbane QLD 4001, Australia

Injecting a less viscous fluid into a more viscous fluid in a Hele-Shaw cell triggers two-dimensional viscous fingering patterns which are characterised by increasingly long fingers undergoing tip splitting and branching events. These complex structures are considered to be a paradigm for interfacial pattern formation in porous media flow and other related phenomena. Over the past five years, there has been a flurry of interest in manipulating these interfacial fingering patterns by altering the physical components of the Hele-Shaw apparatus. In this Focus on Fluids article, we summarise some of this work, concentrating on a very recent study in which the alterations include replacing one of the two bounding plates with an elastic membrane (Ducloué et al., J. Fluid Mech., vol. 826, 2017, R2). The resulting experimental set-up gives rise to a wide variety of novel interfacial patterns including periodic sideways fingers, dendritic-like patterns and short, flat-tipped viscous fingers that appear to resemble molar teeth. These latter fingers are similar to those observed in the printer's instability and when peeling off a layer of adhesive tape. This delightful work brings together a number of well-studied themes in interfacial fluid mechanics, including how viscous and surface tension forces compete to drive fingering patterns, how interfaces are affected by fluid-solid interactions and, finally, how novel strategies can be implemented to control interfacial instabilities.

Key words: fingering instability, Hele-Shaw flows, pattern formation

\section{Introduction}

A Hele-Shaw cell is a simple device that is normally made up of two flat parallel plates held close together with viscous fluid squashed between them. Distinctive patterns are produced when the two fluids are immiscible, separated by a well-defined interface. The key physics behind the pattern formation is that the interface between the two fluids is unstable when the less viscous fluid displaces the more viscous fluid,

\footnotetext{
$\dagger$ Email address for correspondence: scott.mccue@qut.edu.au
} 
with surface tension acting against the instability. This phenomenon is referred to as the Saffman-Taylor instability.

Typically, interfacial instabilities in Hele-Shaw flows are characterised by the capillary number, which is the ratio of viscous to surface tension forces. Linear stability suggests that shorter waves are stabilised by surface tension, while longer waves are unstable. In a rectilinear geometry, for sufficiently low capillary numbers, there is competition between modes that eventually leads to a single steadily propagating Saffman-Taylor finger, whose width is slightly more than half of the channel width. For larger capillary numbers, the flows are complex, with tip splitting and branching structures. The other extensively studied flow configuration involves injecting the less viscous fluid (or air) into a single point. In this radial case, if the flux of injected fluid is constant, then the speed of the interface, and hence the local effective capillary number, decreases in time. The resulting highly branched fingering patterns are truly remarkable.

An exciting contemporary challenge is to understand how viscous fingering patterns can be manipulated and controlled by altering the physical geometry of the Hele-Shaw cell. These issues are important, since in real applications, such as two-phase flow through a porous medium or a pulmonary tube, the geometry is often complex, involving complicated pathways and compliant walls. One simple control strategy is to taper the Hele-Shaw cell so that the top and bottom plates are no longer parallel (Al-Housseiny et al. 2012), while another is to replace the top Hele-Shaw plate with an elastic membrane (Pihler-Puzović et al. 2012). A third variation is to vary the depth of the Hele-Shaw cell by including a uniform rectangular occlusion that lies parallel to the direction of flow. Here, we review the work of Ducloué et al. (2017a), which combines features of tapered plates, elastic membranes and occluded channels to construct an ingenious experimental apparatus that leads to a rich system of interfacial patterns, including novel short, flat-tipped viscous fingers whose properties are very different to their classical analogues. The significance of this work arises from the remarkable interplay between viscous forces, surface tension, depth variations and complex fluid-solid interactions and, furthermore, from how balancing these competing factors allows for control of a wide class of interfacial instabilities.

\section{Overview}

The research undertaken by Ducloué et al. (2017a) was preceded by some key influential studies. The idea of controlling viscous fingering in a Hele-Shaw flow by manipulating time-dependent experimental inputs (such as injection rate or gap thickness) has been explored in some detail (Li et al. 2009). Further control strategies were developed by altering the physical apparatus itself; for example, Al-Housseiny et al. (2012) constructed a tapered Hele-Shaw cell and showed that a negative gradient in depth could inhibit the normal fingering instability for sufficiently low capillary numbers. Similar results were obtained by replacing the Hele-Shaw plates with elastic membranes, as locally the flow is analogous to that in a tapered cell (Pihler-Puzović et al. 2012; Al-Housseiny et al. 2013), and so the geometric mechanism for suppressing the instability is the same.

The experiments of Pihler-Puzović et al. (2012) in a radial geometry motivated Ducloué et al. (2017a) to study a steadily moving front in a converging Hele-Shaw channel, since such a set-up allows for a more controlled study of the unique fingering patterns at the decelerating interface observed by Pihler-Puzović et al. (2012). 
(a)

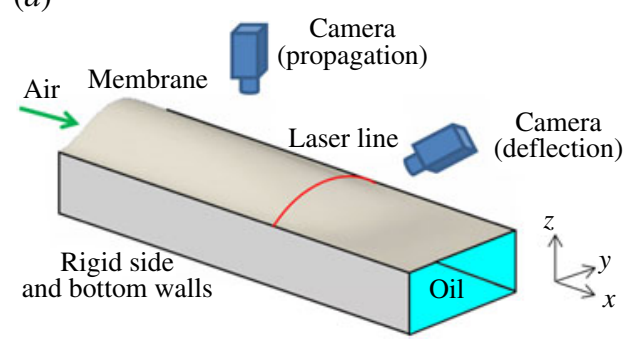

(b)

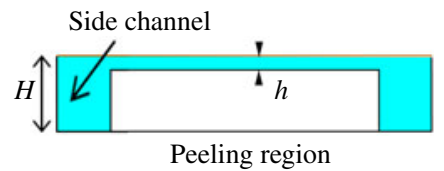

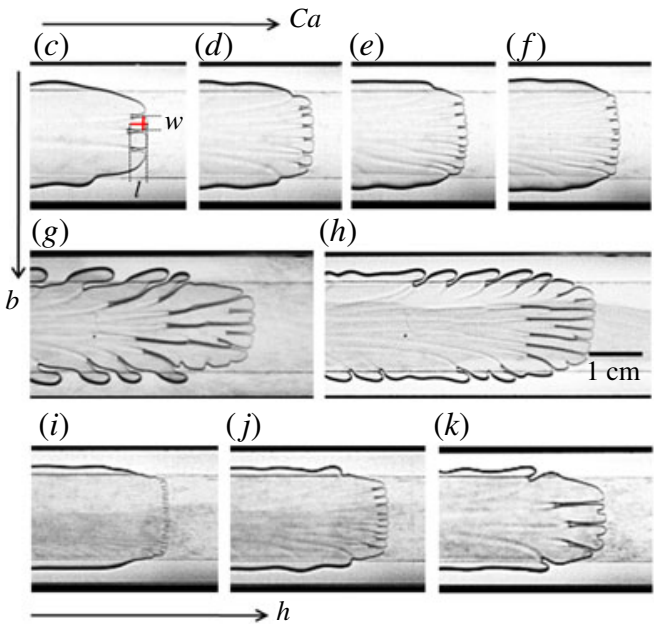

FIGURE 1. (a) Experimental set-up used by Ducloué et al. (2017a), illustrating how air is injected at one end of a Hele-Shaw cell whose top plate is replaced by an elastic membrane. (b) The cross-section showing viscous fluid in blue and a solid uniform rectangular block (the occlusion) in white. $(c-k)$ Typical fingering patterns, where arrows indicate increasing capillary number, $C a$, membrane thickness, $b$, and thickness of the oil layer over the block, $h$. Note the short, flat-tipped, viscous fingers in $(c-f)$ and $(i-j)$ which resemble molar teeth.

The first stage was achieved by the authors in their very recent paper (Ducloué et al. 2017b), where they investigated flow through an initially collapsed channel via a Hele-Shaw cell with an elastic upper boundary. This type of flow geometry is interesting as it has depth gradients in the axial direction and exhibits similar features to that observed when peeling off a strip of viscous adhesive. However, in order to achieve the ideal conditions for studying short, flat-tipped, viscous fingers, one final modification was required, namely, the inclusion of a solid uniform rectangular block that runs along the length of the Hele-Shaw channel, including transverse depth gradients. Ducloué et al. (2017a) refer to this block as an occlusion.

The experimental set-up used by Ducloué et al. (2017a) is illustrated in figure 1(a), with the cross-section of the occluded channel shown in figure 1(b). The width of the shallow central layer of the flow is referred to as the peeling region; its dimensions are controlled by the width of the block. The deep side channels are required in order to maintain a steadily moving almost-flat front.

With these ingredients, Ducloué et al. (2017a) were able to exploit the induced axial and transverse depth gradients to generate a range of fingering patterns along the almost-flat peeling front, examples of which are included in figure $1(c-k)$. These almost-steady fingers have a constant length $l$ and become narrower and shorter as the capillary number increases. Despite the complexity of the underlying flow, the authors determined that the finger length $l$ is inversely proportional to the tangent of the elastic wall's inclination angle; this remarkably simple relationship suggests that, for a given fluid layer thickness, the fingers extend to a constant reopening height. Interestingly, these properties appear analogous to those observed in the viscous adhesive problem as well as the so-called printer's instability (Rabaud et al. 1990). Such short, flattipped, viscous fingers are very different to those normally observed in a Hele-Shaw flow, and in fact look more like molar teeth than fingers! 
(a)

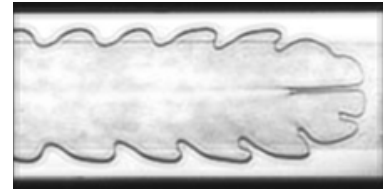

(b)

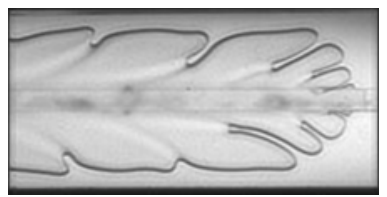

(c)

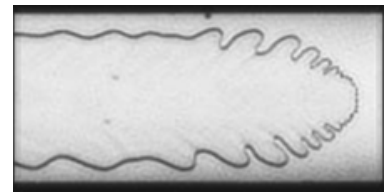

FIGURE 2. The wide variety of interfacial patterns generated by Ducloué et al. (2017a) include spatially periodic sideways oscillations $(a, b)$ and dendritic-like patterns $(c)$.

Other striking fingering patterns are also observed. For example, the flow regimes illustrated in figure 2 do not give rise to a flat peeling front, but instead a curve front emerges. Tip-splitting instabilities appear to drive periodic fingering along the sides of the single long finger (figure $2 a, b$ ). For a very thin rectangular block, dendritic patterns develop (figure $2 c$ ), with very small fingers at the very tip of the long finger. The possibilities for further families of fingering patterns appear endless.

\section{Future}

By extending the work of Ducloué et al. (2017a), there is a plethora of exciting opportunities for studying the connections between various types of viscous fingering patterns. One immediate direction is to explore further the transition from the printer's instability to Saffman-Taylor fingering. Another is to expand the experimental set-up to allow for a broader range of fluid-structure interactions with flexible plates that have more complex constitutive properties than elastic membranes. More generally, there is a need to connect all previously observed viscous fingering patterns, for example via a phase diagram that demonstrates a dependence on geometry, membrane flexibility and flow rate.

Finally, from a modelling perspective, studying viscous fingering in a Hele-Shaw cell provides a number of mathematical challenges. For flow configurations of the type explored in Ducloué et al. (2017a) with elastic membranes and/or occlusions, analytical insight may be sought via depth-averaged models based on the lubrication approximation (Al-Housseiny et al. 2013; Peng et al. 2015).

\section{References}

Al-Housseiny, T. T., Christov, I. C. \& Stone, H. A. 2013 Two-phase fluid displacement and interfacial instabilities under elastic membranes. Phys. Rev. Lett. 111, 034502.

Al-Housseiny, T. T., Tsai, P. A. \& Stone, H. A. 2012 Control of interfacial instabilities using flow geometry. Nat. Phys. 8, 747-750.

Ducloué, L., Hazel, A. L., Pihler-Puzović, D. \& Juel, A. $2017 a$ Viscous fingering and dendritic growth under an elastic membrane. J. Fluid Mech. 826, R2.

Ducloué, L., Hazel, A. L., Thompson, A. B. \& Juel, A. $2017 b$ Reopening modes of a collapsed elasto-rigid channel. J. Fluid Mech. 819, 121-146.

Li, S., Lowengrub, J. S., Fontana, J. \& PalfFy-Muhoray, P. 2009 Control of viscous fingering patterns in a radial Hele-Shaw cell. Phys. Rev. Lett. 102, 174501.

Pihler-Puzović, D., Illien, P., Heil, M. \& Juel, A. 2012 Suppression of complex fingerlike patterns at the interface between air and a viscous fluid by elastic membranes. Phys. Rev. Lett. 108, 074502.

Peng, G. G., Pihler-Puzović, D., Juel, A., Heil, M. \& Lister, J. R. 2015 Displacement flows under elastic membranes. Part 2. Analysis of interfacial effects. J. Fluid Mech. 784, 512-547.

Rabaud, M., Michalland, S. \& Couder, Y. 1990 Dynamical regimes of directional viscous fingering: spatiotemporal chaos and wave propagation. Phys. Rev. Lett. 64, 184-187. 